\title{
Optimisation approaches to constraint satisfaction problems in computer vision
}

\author{
Kazuhiko Yamamoto \\ Electrotechnical Laboratory \\ Tsukuba, Ibaraki 305 \\ JAPAN \\ yamamoto@etl.go.jp
}

\begin{abstract}
This paper describes several new image understanding methods based on parallel operation. There are several constraint satisfaction approaches using a energy minimization. We show how we reconstruct three dimensional surfaces from contours without elevation data and sparse points of known elevation data by using this approach. We also introduce Active Net using this approach and apply this model to segmentation and binocular stereo matching. We experimented with these energy minimization approaches to solve the problems of early and intermediate level of computer vision and show some of the results of our recent research.
\end{abstract}

\section{Introduction}

Image understanding problems in the real world are regarded as an inverse problem. Such a problem often becomes an "ill-posed problem" as it can not be solved analytically. In the field of computer vision, there have been many attempts to model and simulate various aspects of the human vision system. However, some of these lack a theoretical basis. Recently, the energy minimization approach has become one of the principal image understanding techniques as it has been able to provide a common mathematical basis for problems of early and intermediate level vision.

There are several energy minimization approaches such as neural networks, stochastic relaxation, regularization, simulated annealing and other parallel methods [1]. A lot of constraint satisfaction approaches [2] exists in the image understanding field as shown in Figure 1. The regularization method involves the minimization of some energy function which is defined as the sum of the penalty function and of the stabilizer function. The penalty function is the constraint that describes the conformity of the information extracted from the image. The stabilizer function describes the constraint which applies to the whole solution space and frequently takes the form of a smoothness constraint. There have been a number of attempts to solve this kind of problem using the regularization method which changes an ill-posed problem into a well-posed problem by imposing some constraints on the solution space. 


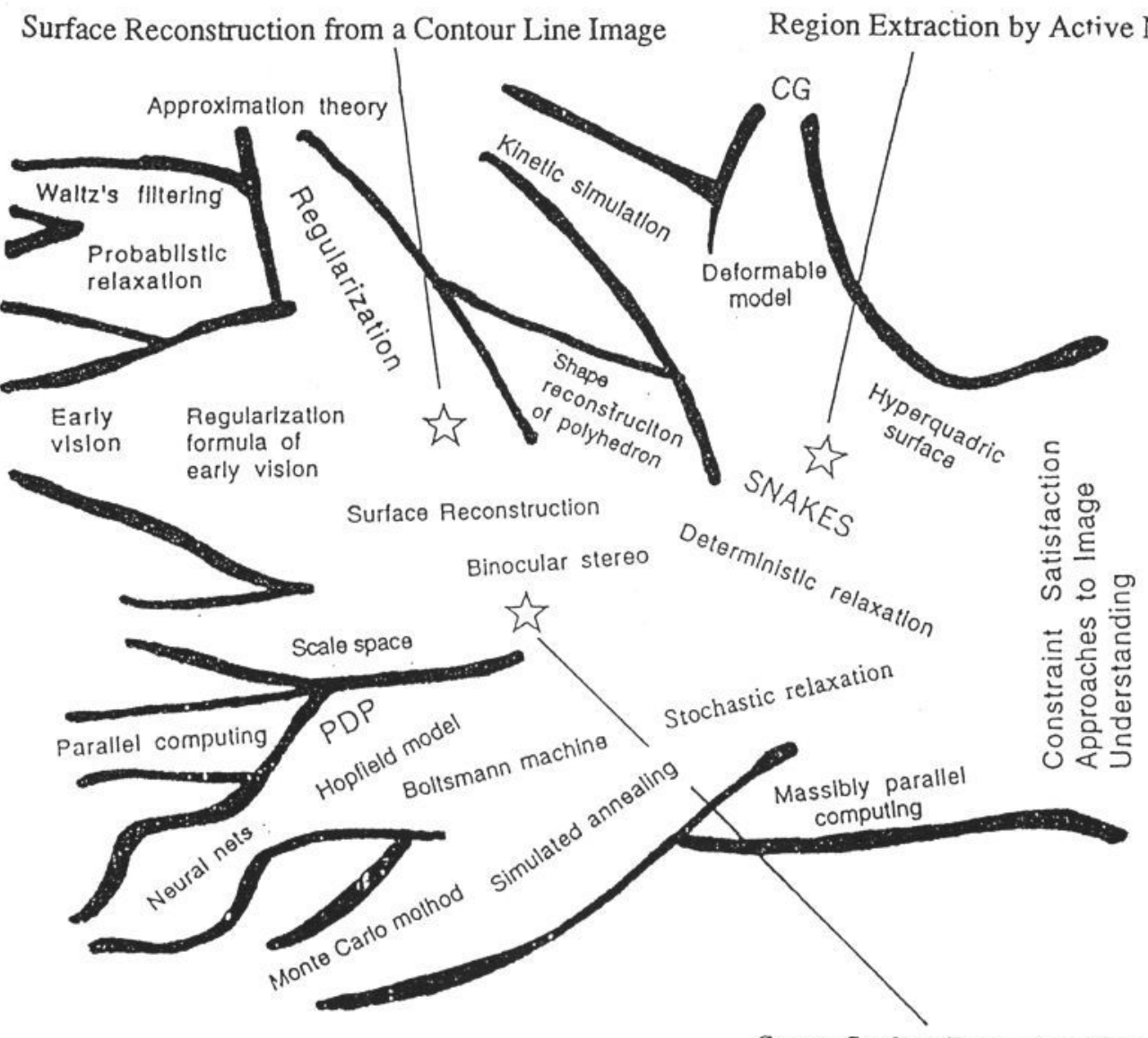

Stereo Surface Reconstruction

Figure 1: Stream of energy minimization approach.

\section{Three dimensional surface reconstruction}

We reconstruct three dimensional surfaces from sparse elevation data and dense contours without using elevation values of topographic map. If we knew the elevation of each contour line, this problem could be solved by a simple transformation between three-dimensional models. Contour lines in actual maps are often broken making the tracking of contour lines difficult. It then becomes necessary to restore contour lines by examining neighboring contour lines.

\section{$2.1 \quad$ Formulation}

Regularization is an approach that avoids the above problems. We define three different constraints on reconstruction evaluation of the value $u$ of the desired surface function over a given area of size $m \times n$. Surface reconstruction using contour line information is performed by minimizing the following energy function: 


$$
E=E_{\text {smooth }}+\lambda E_{\text {data }}+\mu E_{\text {contour }},
$$

where $\lambda$ is the weight parameter of the penalty function and $\mu$ the weight parameter controlling the strength of the contour line constraint. $E_{\text {data }}$ is used as a penalty function which uses the discrepancy between given sparse elevation data $v$ and the surface function $u$.

$$
E_{\text {data }}=\sum_{i=1}^{m} \sum_{j=1}^{n} S_{(i, j)}\left[u_{(i, j)}-v_{(i, j)}\right]^{2},
$$

which is used as a penalty function. Here $S_{(i, j)}$ has the value of 1 at the point where the evaluation values are given and the value of 0 otherwise.

Although the contour lines extracted from topographic maps do not have elevation values, we can obtain the directional components $(\Delta x, \Delta y)$ of contour lines. A picture generated by setting the line of sight to be horizontal would have contour lines that are seen as straight parallel lines. We introduce another penalty function $E_{\text {contour }}$ for a contour line constraint. The elevation value for all pixels on the same contour line should keep same value. For this constraint, we use a function:

$$
E_{\text {contour }}=\sum_{i=1}^{m} \sum_{j=1}^{n}\left[\frac{\partial u}{\partial x} \Delta x_{(x, y)}+\frac{\partial u}{\partial y} \Delta y_{(x, y)}\right]^{2},
$$

which should be minimized for all contour line pixels [4]. Because the summation is carried out for all image pixels, the values $\Delta x$ and $\Delta y$ for every pixel which does not fall a contour line are set to 0 .

$E_{\text {smooth }}$ is used as a smoothness constraint. The quadratic function:

$$
\begin{aligned}
& E_{\text {smooth }} \\
& =\iint_{\Omega}\left[\left(\frac{\partial^{2} u}{\partial x^{2}}\right)^{2}+2\left(\frac{\partial^{2} u}{\partial x \partial y}\right)^{2}+\left(\frac{\partial^{2} u}{\partial y^{2}}\right)^{2}\right] d x d y,
\end{aligned}
$$

is used as a stabilizer function [3].

\subsection{Evaluation}

The extraction of contour lines was done by choosing pixels that are close to the color used for representing contour lines. After thinning and removing branch points, the lines with length less than 2 pixels were considered as noise and ignored [4]. In order to assign directional cosines, we used the value 1 for $\lambda$ and the value 1 for $\mu$ of equation 1 . We tried to reconstruct the topography using the texture of the contours shown in Figure 2.

We presented a method for reconstruction of a 3D-surface from a contour image without relying on dense elevation values. Complicated surfaces were reconstructed by the simple procedure of minimizing the energy function defined for the image. The contour line constraint was derived by considering only the horizontal nature of contour lines, and the regularity of their elevation values was neglected. We have successfully applied this method to topographic maps and got good results as shown in Figure 3. 


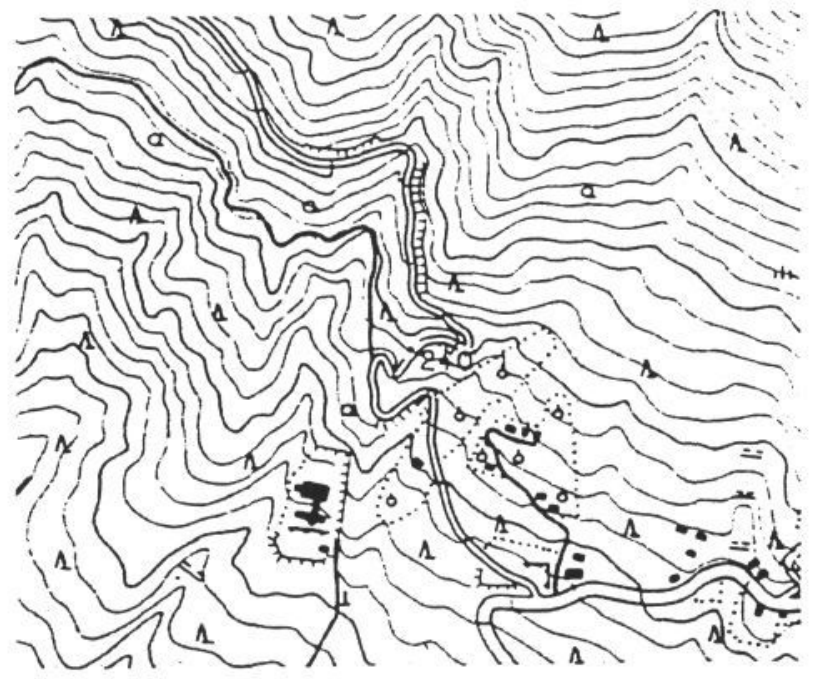

Figure 2: A topographic map.

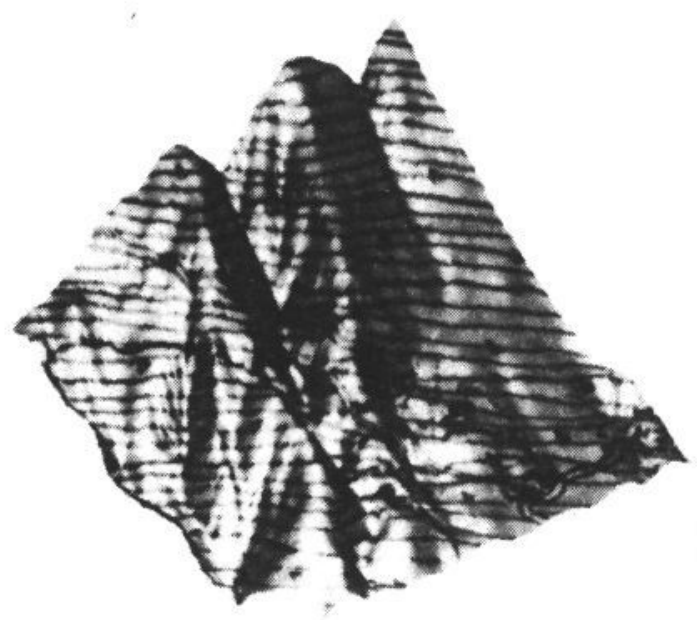

Figure 3: Reconstructed topography result. 


\section{Active Net}

Active Net [6] is a simulated two dimensional elastic network model which shows both active and dynamic behavior. The energy function of the Active Net has two parts ; an internal strain energy of the network and an image energy which attracts the Active Net towards the image features. The network deforms its shape so as to minimize partially the energy function. There are two types of Active Net are radial shape and square shape.

\subsection{Framework}

The position of Active Net is represented by the positions of its nodes. We can represent the nodes parametrically as

$$
v_{(p, q)}=\left(x_{(p, q)}, y_{(p, q)}\right)
$$

where $0 \leq p \leq 1,0 \leq q \leq 1$. Each node connects with four neighboring points:

$$
v(p, q-l), v(p-k, q), v(p+k, q), v(p, q+l),
$$

where $k=\frac{1}{N_{p}}, l=\frac{1}{N_{q}}$, and $N_{p}$ is the number of node points along the p parameter and $N_{q}$ is the number of node points along the q parameter. In a similar manner to the Snakes[7] approach, we can express the energy function of the Active Net as

$$
E_{n \in t}^{*}=\int_{0}^{1} \int_{0}^{1}\left(E_{\text {int }}(v(p, q))+E_{\text {image }}(v(p, q))\right) d p d q
$$

where $E_{\text {int }}$ represents the internal strain energy of the Active Net and $E_{\text {image }}$ represents the image energy which attracts the Active Net towards the image features.

As an extension of the Snakes approach, the internal constraint energy of the Active Net can be written as

$$
\begin{aligned}
& E_{i n t}=\left(\alpha\left(\left|v_{p}\right|^{2}+\left|v_{q}\right|^{2}\right)+\beta\left(\left|v_{p p}\right|^{2}+\left|v_{p q}\right|^{2}+\left|v_{q q}\right|^{2}\right)\right) / 2 \\
& v_{p q}=\frac{\partial^{2} v}{\partial p \partial q}
\end{aligned}
$$

where $\alpha$ and $\beta$ are constants to control the relative importance of the two terms in $E_{\text {int }}$. The first-order term $\left|v_{p}\right|^{2}+\left|v_{q}\right|^{2}$, as shown in Figure 4, represents the force that contracts the Active Net in size and the second-order term $\left|v_{p p}\right|^{2}+$ $\left|v_{p q}\right|^{2}+\left|v_{q q}\right|^{2}$, as shown in Figure 5, represents the force that minimizes the change of gradient between a node and its neighboring node.

\subsection{Image Energy}

In order to apply Active Net to region extraction, an energy function is required to attract the network to a target region in an image. For example, we can use image intensity as the simplest image energy function as follows:

$$
E_{\text {image }}=w I(x, y)
$$




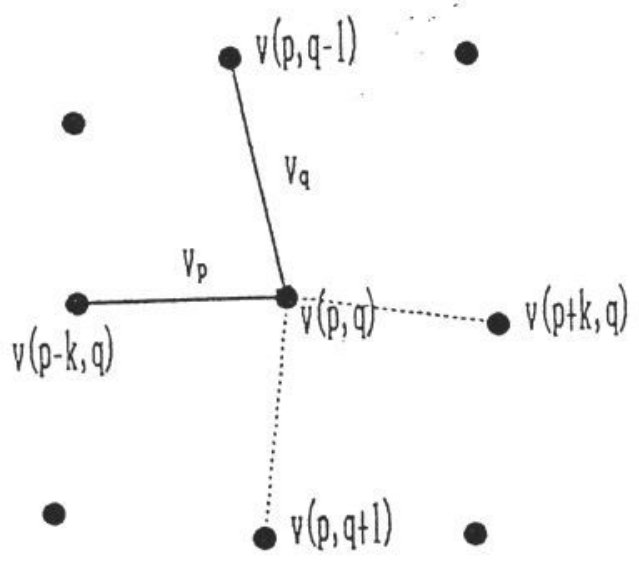

Figure 4: The first-order term.

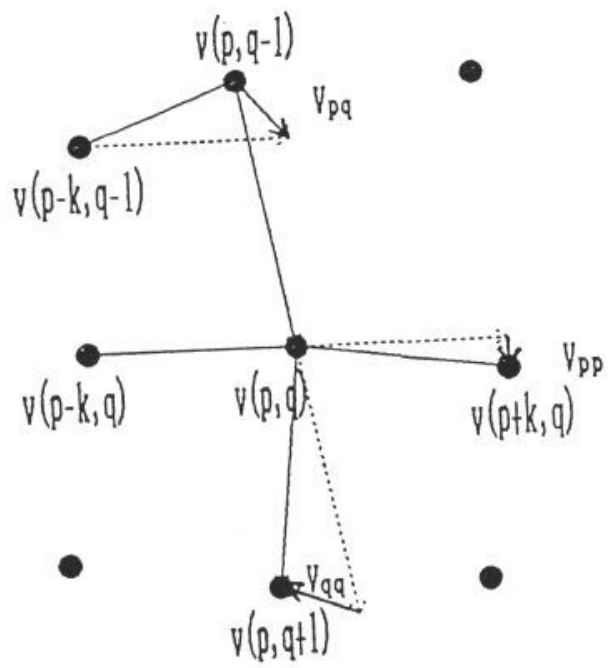

Figure 5: The second-order term. 
where $w$ represents a coefficient and $I(x, y)$ represents the intensity of a point $(x, y)$ in the image. According to the sign of $w$, the network will be attracted either to a bright or dark region. When $w$ is negative, the network is attracted to a bright region and when $w$ is positive, it is attracted toward a dark one.

\subsection{Segmentation}

By using energy minimization, it is possible to wrap the target region within the network, as shown in Figure 6. To enable the nodes on the perimeter of the Active Net to snap to the edge of a target region, we give a boundary condition to the coefficient $w$ in equation 9 . When the Active Net precisely wraps to the target region, the energy function is minimum.

In Figure 6, the initial network which has a radial shape deforms its shape toward the target region. Eventually, the Active Net wraps around the target region and fits itself perfectly to the concave part of the object. Its energy function consists of the internal strain energy of the network and the image energy which attracts the network to features of a target region. We have managed to successfully apply the Active Net to the extraction of a textured region [9].

\subsection{Stereo Matching}

Computational stereo is a method for recovering information concerning the threedimensional shapes of objects by comparing two images of a scene from different perspectives. Once two stereo images are brought into point-to-point correspondence, we can obtain range values by trigonometric means. Another advantage of computational stereo is that the method is passive. In recent years, many authors have been interested in the use of energy minimization approaches for image processing [1] and recently this approach has been applied to stereo matching $[7,8]$.

For stereo matching, we used the square type Active Net. A pair of networks is spread on both right and left stereo images. The networks deform their shapes flexibly so as to minimize the energy function until node-to-node stereo correspondence is completed, and the optimal distribution is obtained. A pair of networks is spread on the right and left stereo images. Each network has its own energy function which consists of the image energy to evaluate the correspondence of the right and left image and the internal strain energy. Since we need no force to contract the Active Net in size in the case of stereo application, the internal constraint energy of the Active Net can be written as follows.

$$
E_{\text {int }}=\beta\left(\left|v_{p p}\right|^{2}+\left|v_{p q}\right|^{2}+\left|v_{q q}\right|^{2}\right) / 2
$$

This constraint effectively forms a disparity smoothness constraint which is often employed in stereo matching. The stereo matching by Active Net is completed when a corresponding pair of nodes of the right and left networks represents a corresponding pair of points in the right and left images. Assuming that the corresponding points in right and left images have the same gray level, we can define the image energy function as follows.

$$
E_{\text {image }}=w\left|I_{R}(u(p, q))-I_{L}(v(p, q))\right|^{2}
$$




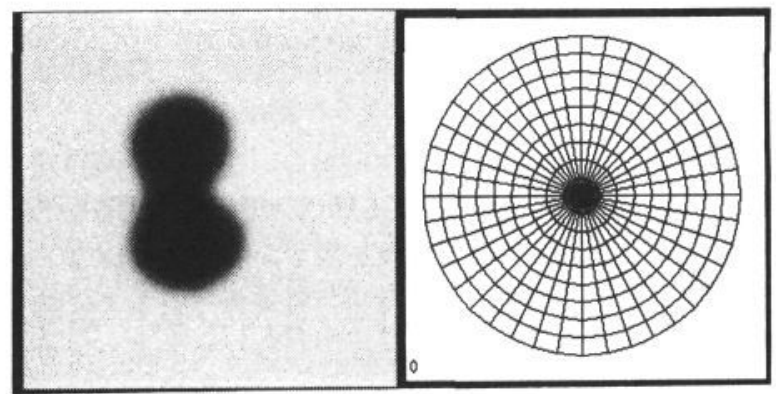

(a)

(b)

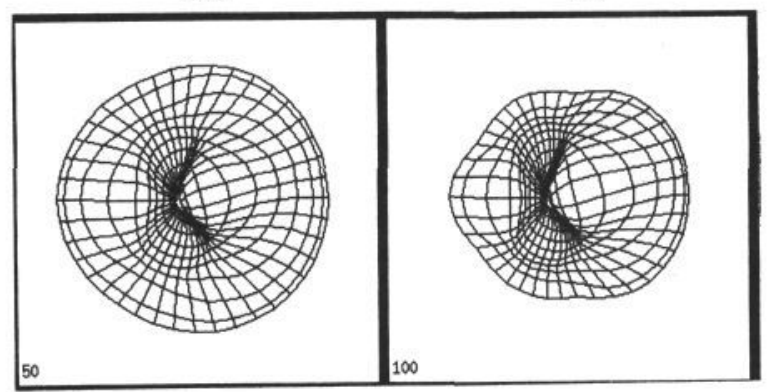

(c)

(d)

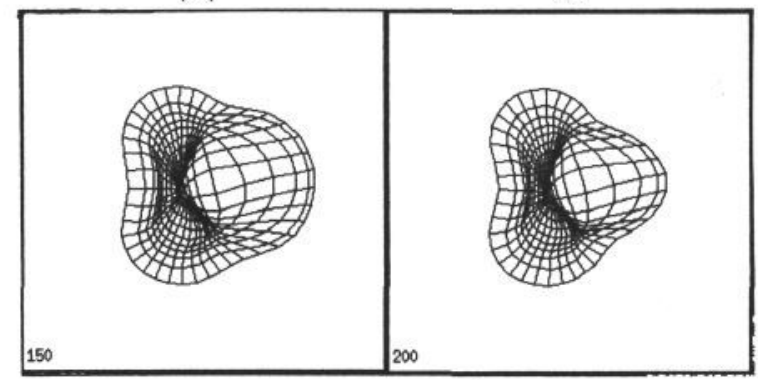

(e)

( f )

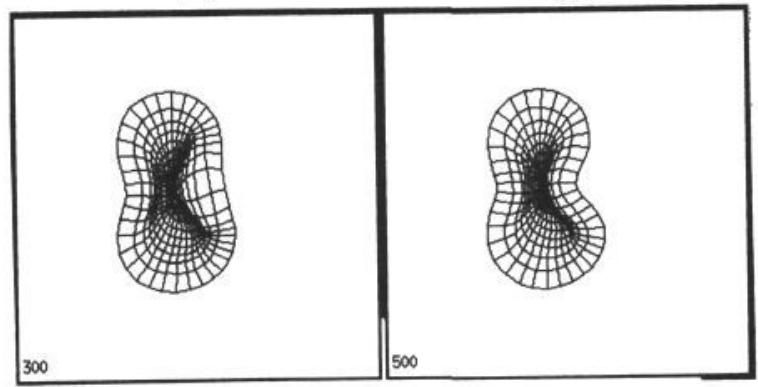

(g)

(h)

(a)The input pattern, (b) the initial net, (c)-(h) iteration.

Figure 6: Radial Active Net applied to a test pattern. 
where $I_{R}(u(p, q))$ represents the gray level at the location of the right-network node point $u(p, q)$ and $I_{L}(v(p, q))$ represents the gray level at the location of the left-network node point $v(p, q) ; w$ is a weight coefficient. The energy function to be minimized in the stereo Active Net is as follows.

$$
E_{n \in t}^{*}=\int_{0}^{1} \int_{0}^{1}\left(\beta\left(\left|v_{p p}\right|^{2}+\left|v_{p q}\right|^{2}+\left|v_{q q}\right|^{2}\right) / 2+w\left|I_{R}(u(p, q))-I_{L}(v(p, q))\right|^{2}\right) d p d q
$$

We wish to obtain a solution to the energy function of equation 12. Two energy functions for right and left images can be defined as follows.

$$
\begin{gathered}
R E_{n e t}^{*}=\int_{0}^{1} \int_{0}^{1}\left(E_{\text {int }}(u(p, q))+E_{\text {image }}(u(p, q))\right) d p d q \\
=\int_{0}^{1} \int_{0}^{1}\left(\beta\left(\left|u_{p p}\right|^{2}+\left|u_{p q}\right|^{2}+\left|u_{q q}\right|^{2}\right) / 2+w\left|I_{R}(u(p, q))-I_{L}(v(p, q))\right|^{2}\right) d p d q(13) \\
L E_{n e t}^{*}=\int_{0}^{1} \int_{0}^{1}\left(E_{\text {int }}(v(p, q))+E_{\text {image }}(v(p, q))\right) d p d q \\
=\int_{0}^{1} \int_{0}^{1}\left(\beta\left(\left|v_{p p}\right|^{2}+\left|v_{p q}\right|^{2}+\left|v_{q q}\right|^{2}\right) / 2+w\left|I_{R}(u(p, q))-I_{L}(v(p, q))\right|^{2}\right) d p d q(14)
\end{gathered}
$$

\subsection{Experimental Results}

We have applied our method to real and random dots stereo images. Our simultaneous equations were often trapped in local minima because of the local roughness of the image. To avoid this problem, we introduced a scale-space strategy [10] which sets a coarse surface at initial situation, and avoids getting caught in a local minimum, and then to change the surface fine. We employed three stage minimization from coarse to fine which were made by three different sizes of Gaussian filters $(r=3.0,5.0,9.0)$. We applied the energy minimization to the rough image and converged on a network. Next, we used this result as input of the succeeding minimization process with a fine image. We repeated this process three times so as to converge near the optimum. In Figure7, bottom shape shows the result of stereo matching by Active Net applied to top images.

\subsection{Unknown epipolar}

In the case of these stereo pairs, epipolar lines are parallel to the $\mathrm{x}$ axis (horizontal axis) so that equation 12 is not used to move the nodes in the $y$-direction. The calculated disparity distribution is shown by a gray scale image in which brightness is proportional to disparity. The geometry of the imaging system is usually unknown; thus we can not employ the epipolar constraint and we have to include equation 12 which allows the nodes of the networks to move in both the $\mathrm{x}$ and $\mathrm{y}$ directions. Results for artificial random pattern stereo pairs are shown in Figure 8. There is a vertical displacement of the inner rectangular area in Figure 8, even though we can still reconstruct the original shape. 

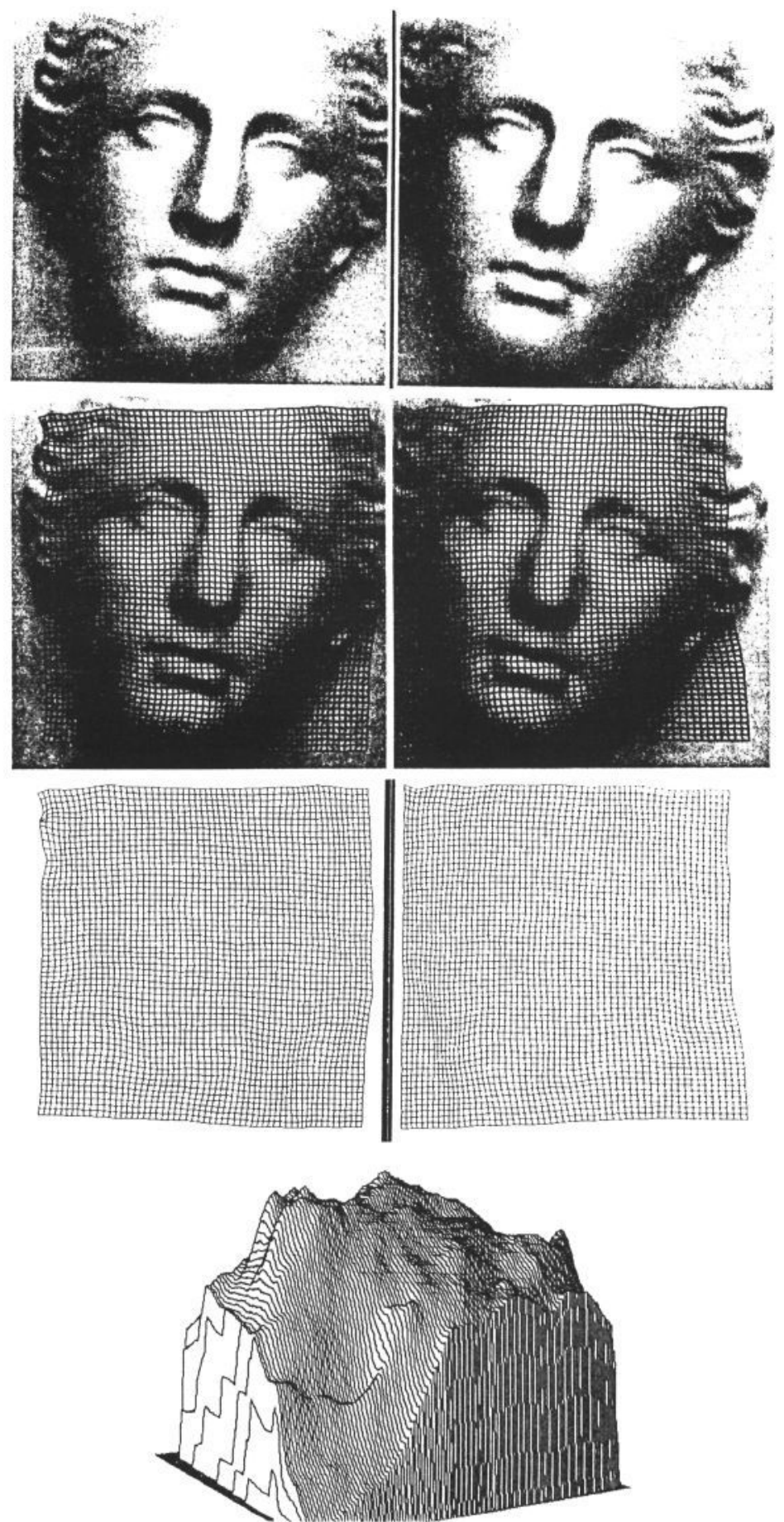

Figure 7: Stereo matching applied to real stereo pair(Venous). From top to bottom, the initial net, the converged net (with and without the original stereo images), bird-view display. 

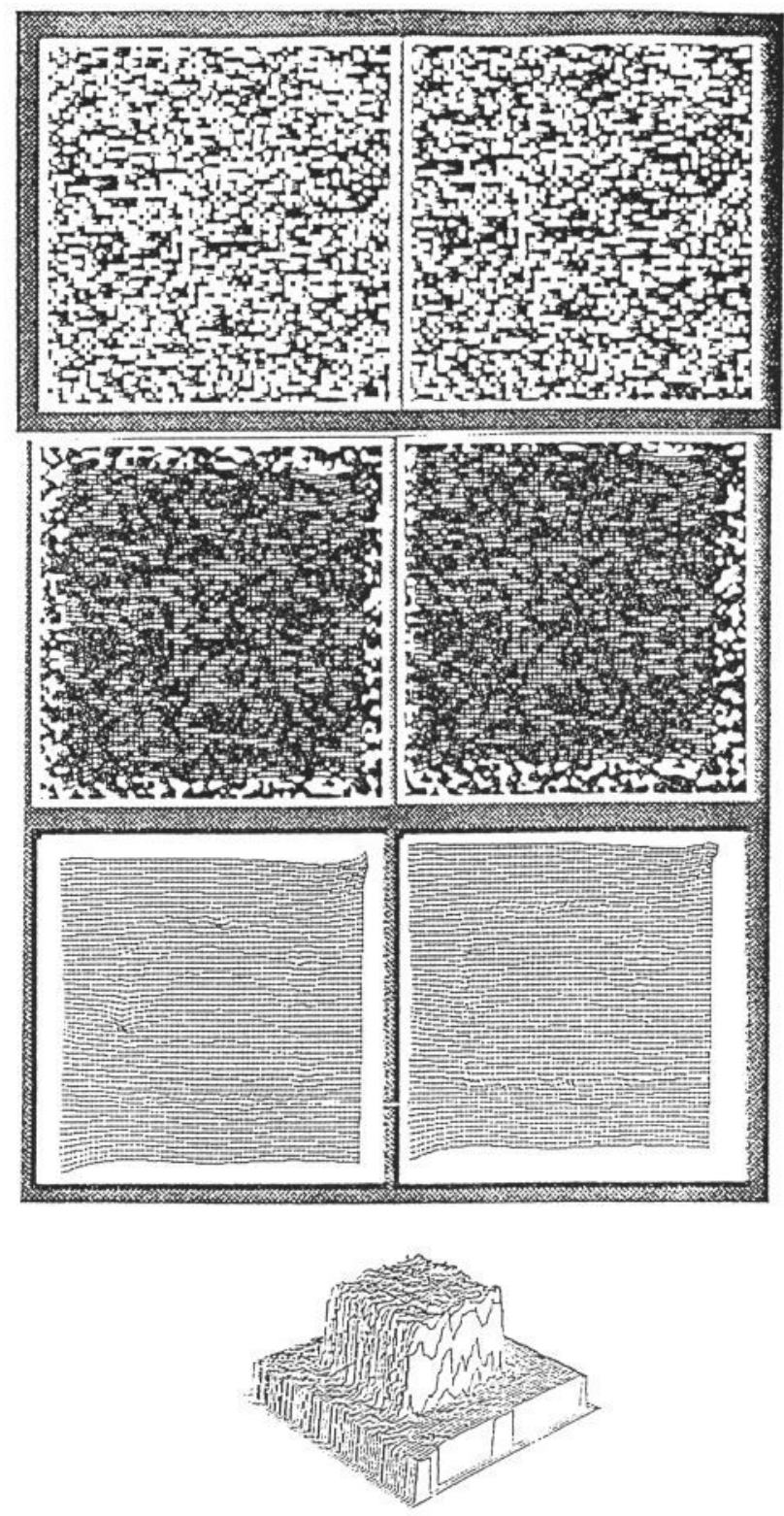

Figure 8: Stereo matching applied to a random pattern stereo pair. From top to bottom, stereo pair, initial net, the converged net, bird-view 


\section{Shape description of range data}

We introduce the "blobby model" for automatically generating a shape description from range data. This method expresses a surface of an object as an isosurface of a scalar field which is generated from field generating primitives [11]. To determine the distribution of primitives, an energy function which measures the shape difference between the range data and the nodel is minimized. We start with a single primitive and introduce more primitives by splitting each primitive into further primitives so as to reduce the energy value. In this manner, the shape of the $3 \mathrm{D}$ object is slowly recovered as the isosurface produced by many primitives. We have successfully applied this method to range data of some human faces.

\section{Conclusion}

These approaches are fit for early and intermediate vision. There is an open problem to combine top down information and the bottom up information. We are currently trying to introduce an attention method to solve this problem [5]. Another current research involves combining Genetic Algorithm and Active Net for stereo matching. It involves using the Active Nets found using the previous method as individuals for the Genetic Algorithm. Those methods consume a lot of computation time on ordinary computers but are suitable for parallel implementation.

\section{Acknowledgment}

This work was supported by the "Real World Computing Program" of the MITI. Author thanks Dr.Hiromitsu Yamada, Dr. Katsuhiko Sakaue and Dr. Sigeru Muraki for cooperation research, and Dr. Nobuyuki Otsu for his support during this work.

\section{References}

[1] Poggio T., Torre V. and Koch C. Computational Vision and Regularization Theory. Nature, vol.317, no.6035, pp.314-319, 1985.

[2] Yamamoto K. Constraint Satisfaction Approaches to Image Understanding RWC Technical Report, TR-94001,pp.7-8,1994.

[3] Grimson W. An implementation of a computational theory of visual surface interpolation Computer.Vision,Graphics, Image Processing, vol. 22, pp.39-69, 1983.

[4] Muraki S., Yokoya N., Yamada H. and Yamamoto K. Reconstruction of a 3D Surface maintaining consistency with contour lines Proc. of Int. Conf. Image Processing, pp.203-206, 1989. 
[5] Hasegawa O. and Yamamoto K. Recent trend on human vision and computer vision Proc. 8th Symposium on Biological and Physiological Engineering,pp.27,1993(in Japanese).

[6] Sakaue K. and Yamamoto K. Active Net model for stereo matching ETL Technical Report, TR-90-49, Electrotechnical Laboratory, 1990.

[7] Kass M., Witkin A. and Terzopoulos D. Snakes: Active Contour Models International Journal of Computer Vision, vol.1, no.4, pp.321-331, 1988.

[8] Yokoya N. Surface Reconstruction Directly from Binocular Stereo Images by Multiscale-Multistage Regularization 11th ICPR, pp.642-646, 1992.

[9] Tsumiyama Y., Sakaue K. and Yamamoto K. Active Net : Active Network Model for Region Extraction The 39th National Conference of Information Processing Society of Japan, 2E-4, 1989 (in Japanese).

[10] Witkin A., Terzopoulos D. and Kass M. Signal Matching Through Scale Space International Journal of Computer Vision, vol.1, no.2, pp.133-144, 1987.

[11] Muraki S. Volumetric shape description of range data Computer Graphics, vol.25, no.4, pp.227-235, 1992. 
